\title{
GeneXpert MTB-RIF assay as a rapid and effective molecular method for the diagnosis of tuberculosis in Erbil City
}

Accepted: 19/11/ 2018

Salah Tofik J alal Balaky ${ }^{1 *}$

\section{Abstract}

Background and objective: Tuberculosis is a major public health problem worldwide, especially in developing countries. The disease poses a massive threat to public health, causing increased morbidity and mortality. Therefore, early detection is essential to reduce the death rate and interrupt transmission. In this study, GeneXpert assay was used for the rapid identification of bacteria, and its sensitivity and specificity were compared with acid fast bacilli staining in both pulmonary and extrapulmonary clinical specimens.

Methods: A total of 115 pulmonary and extrapulmonary samples were collected and diagnosed for tuberculosis detection. Acid fastbacilli, culture, and GeneXpert methods were used for the diagnosis of tuberculosis.

Results: Out of 115 tuberculosis specimens, 70 were acid fast bacilli smear positive, while 97 samples were positive for Mycobacterium tuberculosis by GeneXpert assay. The results indicated a higher sensitivity of GeneXpert assay compared to the acid fastbacillistaining method. The sensitivity of GeneXpert was $91.6 \%$, specificity $50 \%$; positive and negative predictive values were $89.7 \%$ and $55.6 \%$, respectively. No significant differences were detected between the Gene Xpert test results and the culture results $(P=0.815)$, and the total agreement between the tests was $84.3 \%$.

Conclusion: We concluded that the GeneXpert test results is sensitive as culture results for both pulmonary and extrapulmonary specimens. Although culture remains the gold standard for laboratory confirmation of tuberculosis disease, GeneXpert should become standard practice for patients suspected of tuberculosis, and all clinicians and public health tuberculosis programs should have access to molecular testing for tuberculosis to shorten the time of diagnosis.

Keywords: Mycobacterium tuberculosis; Diagnosis; Pulmonary; Extrapulmonary; GeneXpert; AFB.

\section{Introduction}

Tuberculosis (TB) remains one of the major infectious diseases, and is a global public health problem, especially in developing countries. The disease poses a massive threat to public health. In 2015, there were about 10.4 million TB cases in the world, from which $90 \%$ were adults. ${ }^{1}$ The TB causative pathogen, Mycobacterium tuberculosis (M. tuberculosis), continuously produces strains harboring resistance genes, thus causing increasing challenge to TB treatment and prevention. ${ }^{2}$ $M$. tuberculosis one of the leading causes of death from infectious diseases and is infected about $25 \%$ of the world's population. ${ }^{3}$ Initially, TB attacks the lungs; however, other organs can be attacked by the pathogen, including the lymphatic system, blood circulation, central nervous system, and alimentary system. ${ }^{4}$ It has been reported that in Iraq, more than 8000 TB cases were notified in 2015 with an incidence of $16 / 100,000$ population. ${ }^{5}$ The failure to act quickly in recognizing and treating TB patients leads to increased mortality, secondary resistance, and continuous transmission. ${ }^{6}$ Although smear

${ }^{1}$ Department of Medical Microbiology, College of Health Sciences, Hawler Medical University, Erbil, I raq.

* Correspondence: sbalaky2013@gmail.com 
microscopy and radiography are rapid and inexpensive, they have poor sensitivity and a poor positive predictive values, however, they remained as a valuable diagnosis in most resource-constrained settings. ${ }^{7}$ As in developing countries, TB diagnosis still relies mainly on the use of smear microscopy, which has a low sensitivity and specificity compared to the culture. The microbiological methods for TB diagnosis by culture remains the gold standard. ${ }^{8}$ Culture is the "gold standard" for final determination, but it is slow and may take up to eight weeks because of the pathogen long generation time. ${ }^{9}$ Thus a rapid and accurate method for the diagnosis of $M$. tuberculosis is essential for earlier treatment initiation, its prevention, improved patient outcomes, and more effective public health interventions. ${ }^{10}$ The demand and the urgent need for a rapid and simple diagnostic test, particularly in developing countries. A PCR based technique for TB detection and antibiotic resistance testing was developed. ${ }^{11}$ GeneXpert assay is a fully automated molecular test for the diagnosis of M. tuberculosis, and it can detect resistance to rifampin (RIF). The test is a Real-Time PCR assay for amplification of a specific sequence of the $r p o B$ gene of the pathogen. The gene is probed with molecular beacons to investigate mutations within the rifampin-resistance determining region. ${ }^{12}$ The technique has been approved by the Scientific Board of the World Health Organization (WHO) as the most sensitive and rapid test for TB diagnosis, and it has much better accuracy than sputum smear microscopy. ${ }^{1}$ This on-demand assay takes less than two hours to be performed, and it includes bacterial lysis, DNA extraction, amplification, and amplicon detection using a disposable plastic cartridge. ${ }^{13}$ In addition to that, the assay has a high sensitivity, low complexity, minimum hands-on, and reduced safety concerns for laboratory staff members. ${ }^{14,15}$ This study aimed to investigate the diagnostic accuracy of GeneXpert assay in pulmonary and extrapulmonary clinical specimens and to compare it with acid fact bacilli (AFB) taking culture as a reference test in Erbil city.

\section{Methods}

\section{Study design and sample collection}

This diagnostic accuracy study was conducted from June to August 2018, in suspected patients of pulmonary and extrapulmonary tuberculosis, at the Chest and Respiratory Disease Specialized Centre in Erbil City. A total of 115 samples (44 pulmonary and 71extrapulmonary) were collected from patients with symptoms suggestive of TB disease. Suspected TB patients for collecting specimens were assigned in this study on the bases of presenting symptoms and chest radiography findings. Pulmonary specimens were taken from the sputum. Patients meeting the clinical eligibility criteria were asked to provide three sputum specimens over a two day period, two spot samples and one obtained in the morning. Extra-pulmonary specimens were taken from pleural fluid, lymph node biopsy, gastrointestinal, cerebrospinal fluid, skin, genitourinary. Nonsterile clinical specimens were processed by the conventional $\mathrm{N}$-acetyl-Lcysteine-NaOH method. ${ }^{15}$ The study protocol was reviewed and approved by the Scientific and Research Ethics Committee of the College of Health Sciences, Hawler Medical University.

\section{AFB and Culture}

Collected samples were processed in a special laboratory in TB center, then two of the three samples were randomly selected and processed with $\mathrm{N}$-acetylcysteine and sodium hydroxide (NALC$\mathrm{NaOH}),{ }^{16}$ followed by centrifugation. The samples were resuspended in $1.5 \mathrm{~mL}$ of sample buffer and subjected to microscopy with Ziehl-Neelsen staining and inoculated on solid medium (Löwenstein- Jensen, bioMerieux, France). ${ }^{17}$ The third sputum sample was tested directly by ZiehlNeelsen microscopy and the GeneXpert test. Solid cultures were considered 
negative after 42 days of incubation without isolation of any mycobacteria. Non-respiratory specimens from closed and normally sterile sites were not decontaminated prior to smear preparation and culture but were concentrated by centrifugation at 3,000 $\mathrm{g}$ for 20 minutes. Processed specimens from nonsterile sites and centrifuged specimens from sterile sites were directly cultured. Conventional methods and clinical symptoms that strongly indicate the presence of mycobacteria and/or positive bacilloscopy for AFB were used as criteria for the confirmatory culture of patient's samples. ${ }^{18}$ The positive culture was further identified as MTB by MGIT TBc identification, which is used for the identification of the $M$. tuberculosis complex from MTB culture. $^{18}$ The TBc ID test is a lateral-flow immune-chromatographic assay based on the detection of MPB64 in cultures using an MPT64-specific monoclonal antibody (Becton Dickinson, USA).

\section{GeneXpert assay}

The GeneXpert assay was used as previously used. ${ }^{15}$ Briefly, the provided buffer was added at a 3:1 ratio to clinical samples. The tubes were mixed manually twice in 15-min period at room temperature before $2 \mathrm{~mL}$ of the inactivated material was transferred to the test cartridge. The cartridge was then inserted into the test platform, and the hand on work ended. Then the machine automatically filtered, washed, and ultrasonically lysed to release DNA. Real-time PCR amplification and detection were performed in an integrated reaction tube. Primers used for this assay were forward:

(CGTGGAGGCGATCACACCG CAGAC) and reverse:

\section{(AGCTCCAGCCCGGCACGCTCACGT)}

(Applied Biosystems). The results were finally read after one hour 45 minutes, in which fluorescent signal was measured automatically. Negative or positive and defined susceptible or resistant to rifampin depending on the detection of mutations in $r p o B$ gene (MTB-RIF Instructions). All specimens that were culture positive and GeneXpert assay negative and specimens that were culture negative and GeneXpert assay positive were retested twice. The last result was used for the analysis.

\section{Statistical analysis}

Data were analyzed using the statistical package for the social sciences (version 22). Frequencies and percentages were calculated. McNemar's test was used (in the 2X2 table) when the results of the AFB test or Gene Xpert test were compared with the culture results (of the same patients); as in the following table:

\section{Culture}

Positive Negative

$P$ (By McNemar)

\begin{tabular}{lcccc}
\hline AFB or Gene Xpert & Positive & TP & FP & TP+FP \\
& Negative & FN & TN & FN+TN \\
& & TP+FN & FP+TN & Grand total \\
Total & &
\end{tabular}

$\mathrm{TP}=$ True positive; $\mathrm{TN}=$ True negative; $\mathrm{FP}=\mathrm{False}$ positive; $\mathrm{FN}=\mathrm{False}$ negative.

Sensitivity = TP / $(T P+F N) * 100 ;$ Specificity = TN / $(F P+T N)^{*} 100 ;$ Predictive value positive $\left(\mathrm{PV}^{+}\right): \mathrm{TP}^{*}$ $(\mathrm{TP}+\mathrm{FP}){ }^{*}$ 100; Predictive value negative $\left(\mathrm{PV}^{-}\right): \mathrm{TN} /(\mathrm{FN}+\mathrm{TN}){ }^{*} 100 ;$ Total agreement $=(\mathrm{TP}+\mathrm{TN}) /$ Grand total.

A $P$ value of $\leq 0.05$ was considered as statistically significant. 


\section{Results}

The study included 115 patients with symptoms suggestive of TB on the bases of clinical, pathological, or radiological evidence of tuberculosis. Their mean age $( \pm \mathrm{SD})$ was $39.64 \pm 18.96$ years, ranging from 17 to 91 years. The median age was 32 years. Table 1 shows that one third of the patients aged 20-29 years and around $10 \%$ aged less than 20 years, while the proportion of those aged 60 years and more was $21.7 \%$. More than half $(54.8 \%)$ of the sample were females, and $58.3 \%$ of the cases of TB were extrapulmonary (Table 1). Table 2 shows that the sensitivity of the sputum AFB test compared with the culture was $69.5 \%$, the specificity was $80 \%$, the positive predictive value $(\mathrm{PV}+)$ was $94.3 \%$, and the negative predictive value (PV-) was $35.6 \%$. The total agreement was $71.3 \%$. Significant differences were detected between the AFB test results and the culture results $(P<0.001)$.

Table 1: Age and gender distribution of the studied sample, and the TB site.

\begin{tabular}{lcc}
\hline Age (years) & No. & $\%$ \\
\hline$<20$ & 11 & 9.6 \\
$20-29$ & 38 & 33.0 \\
$30-39$ & 17 & 14.8 \\
$40-49$ & 10 & 8.7 \\
$50-59$ & 14 & 12.2 \\
$\geq 60$ & 25 & 21.7 \\
Mean $( \pm$ SD) & 39.64 & $( \pm 18.96)$ \\
Gender & & \\
Male & 52 & 45.2 \\
Female & 63 & 54.8 \\
Site & & \\
Pulmonary & 48 & 41.7 \\
Extra-pulmonary & 67 & 58.3 \\
Total & 115 & 100.0 \\
\hline
\end{tabular}

Table 2: AFB test results and indicators of validity compared with the culture as a gold standard.

\begin{tabular}{lcccc}
\hline \multirow{2}{*}{ AFB } & \multicolumn{2}{c}{ Culture } & & Total \\
& Positive & Negative & 70 & \\
\hline Positive & 66 & 4 & 45 & $<0.001$ \\
Negative & 29 & 16 & 115 & \\
Total & 95 & 20 & PV- & Total agreement \\
Sensitivity & Specificity & PV+ & $35.6 \%$ & $71.30 \%$ \\
$69.5 \%$ & $80.0 \%$ & $94.3 \%$ & & \\
\hline
\end{tabular}

*By McNemar's test. 
No significant differences were detected between the GeneXpert test results and the culture results $(P=0.815)$. The total agreement between the tests was $84.3 \%$, as presented in Table 3, which also shows that the sensitivity was $91.6 \%$, the specificity was $50 \%$, the PV+ was $89.7 \%$, and the PV- was $55.6 \%$. Tables 4 and 5 show the diagnostic test analysis among patients with pulmonary TB patients of extrapulmonary TB. The same pattern of the whole sample can be applied for both pulmonary TB and extrapulmonary TB. In Table 4, the results of the AFB test were compared with the results of the culture. Significant differences were detected between the results of the AFB test and the culture among patients with pulmonary and extra-pulmonary TB $(P=0.001$ and $P=$ 0.004 , respectively). The total agreement was $77.08 \%$ for the pulmonary type, and $67.6 \%$ for the extra-pulmonary TB.

Table 3: GeneXpert test results and indicators of validity compared with the culture as a gold standard.

\begin{tabular}{|c|c|c|c|c|}
\hline \multirow{2}{*}{ Gene Xpert } & \multicolumn{2}{|c|}{ Culture } & \multirow[b]{2}{*}{ Total } & \multirow{2}{*}{$P$ value* } \\
\hline & Positive & Negative & & \\
\hline Positive & 87 & 10 & 97 & 0.815 \\
\hline Negative & 8 & 10 & 18 & \\
\hline Total & 95 & 20 & 115 & \\
\hline Sensitivity & Specificity & PV+ & PV- & Total agreement \\
\hline $91.6 \%$ & $50.0 \%$ & $89.7 \%$ & $55.6 \%$ & $84.30 \%$ \\
\hline
\end{tabular}

${ }^{*}$ By McNemar's test.

Table 4: AFB test results and indicators of validity compared with the culture as a gold standard test among patients with pulmonary and extra-pulmonary TB.

\begin{tabular}{|c|c|c|c|c|c|c|}
\hline \multirow[b]{2}{*}{ Site of TB } & \multicolumn{4}{|c|}{ Culture } & \multirow[b]{2}{*}{$\begin{array}{c}\text { Total } \\
\text { agreement }\end{array}$} & \multirow[b]{2}{*}{$P$ value* } \\
\hline & AFB & Positive & Negative & Total & & \\
\hline \multirow[t]{5}{*}{ Pulmonary } & Positive & 30 & 0 & 30 & & 0.001 \\
\hline & Negative & 11 & 7 & 18 & & \\
\hline & Total & 41 & 7 & 48 & & \\
\hline & Sensitivity & Specificity & PV+ & PV- & & \\
\hline & $73.20 \%$ & $100 \%$ & $100 \%$ & 38.9 & $77.08 \%$ & \\
\hline \multirow[t]{5}{*}{ Extrapulmonary } & Positive & 36 & 4 & 40 & & 0.004 \\
\hline & Negative & 18 & 9 & 27 & & \\
\hline & Total & 54 & 13 & 67 & & \\
\hline & Sensitivity & Specificity & PV+ & PV- & & \\
\hline & $66.70 \%$ & $69.20 \%$ & $90 \%$ & $33.30 \%$ & $67.16 \%$ & \\
\hline
\end{tabular}

${ }^{*}$ By McNemar's test. 
Regarding the GeneXpert test (Table 5), no significant differences were detected between the Gene Xpert results and the culture results among patients with pulmonary TB $(P=0.727)$ and patients with extra-pulmonary TB $(P=0.344)$. The total agreement among patients with pulmonary TB was $83.33 \%$, and $85.07 \%$ among patients with extra-pulmonary TB.

\section{Discussion}

The characteristic profile of TB patients in this study showed that the gender predominance among TB patients was seen in females $(n=63 ; 54.8 \%)$ followed by males $(n=52 ; 45.2 \%)$, with their mean of age 39.64. Others reported different ratios in other places around the world. In this regard, our data were in good accordance with what was reported by previous studies, ${ }^{19}$ who reported 57.4 were female with a mean age of 44.2 in their study. The most common age group affected was $20-29$ years $(n=38 ; 33 \%)$ followed by 60 and above $(n=25 ; 21.7 \%)$, these data were found to be different from data produced by Pragya et al., ${ }^{20}$ who reported that 41-60 age group was the most affected one in their study. The conventional methods for the diagnosis of tuberculosis, such as AFB staining method, chest radiography, and culture, have several limitations and are thus not always helpful in TB patient management. ${ }^{1}$ Therefore, there is an urgent need for an effective and rapid diagnostic method. Early diagnosis of TB is extremely important for patient management, preventing bacterial transmission in the community, and effective treatment regimen. ${ }^{21}$ Recent advances in molecular technologies such as GeneXpert assay for the diagnosis of TB in clinical specimens with acceptable turnaround time improved TB control in the world especially in developing countries. ${ }^{22}$ In this study, three methods, including AFB, culture, and GeneXpert methods, were used for the detection of M. tuberculosis in 115 specimens from patients with symptoms suggestive of TB. Sputum smear microscopy for detecting AFB is a rapid, inexpensive, relatively easy to perform, and highly specific tool for identifying persons with active $\mathrm{TB}^{23}$ However, the sensitivity of AFB microscopy was $73.2 \% \quad(30 / 41)$ for culture-positive pulmonary specimens and $66.7 \%$ (36/54) for culture-positive extrapulmonary

Table 5: GeneXpert test results and indicators of validity compared with the culture as a gold standard test among patients with pulmonary and extra-pulmonary TB.

\begin{tabular}{lcccccc}
\hline Site of TB & & \multicolumn{2}{c}{ Culture } & & & $P_{\text {value* }}$ \\
& Gene Xpert & Positive & Negative & Total & Total agreement \\
\hline Pulmonary & Positive & 36 & 3 & 39 & 0.727 \\
& Negative & 5 & 4 & 9 & \\
& Total & 41 & 7 & 48 & \\
& Sensitivity & Specificity & PV+ & PV- & \\
& $87.80 \%$ & $57.1 \%$ & $92.3 \%$ & $44.4 \%$ & $83.33 \%$ \\
Extrapulmonary & Positive & 51 & 7 & 58 & \\
& Negative & 3 & 6 & 9 & \\
& Total & 54 & 13 & 67 & \\
& Sensitivity & Specificity & PV+ & PV- & \\
& $94.40 \%$ & $46.2 \%$ & $87.9 \%$ & $66.7 \%$ & $85.07 \%$ \\
\hline
\end{tabular}


specimens. This agreed with others, who raise concerns about the low sensitivity of AFB staining method for the diagnosis of TB. ${ }^{24,25}$ However, less attention has been given to the fact that AFB smear microscopy may appear positive without actually having mycobacteria. ${ }^{26}$ Statistical analysis showed significant differences between the AFB test results and the culture results $(P<0.001)$. In our study, there were 4 AFB smear positive, while the culture showed negative results. The false negative AFB cases (29/95) that confirmed by culture positive can be attributed to the moderate sensitivity for AFB such that it needs 6000 to 10,000 organisms per $\mathrm{mL}$ of the specimen to give a correct positive result. ${ }^{15}$ While the threshold for culture to be positive is only 100 bacilli per $\mathrm{mL}$ of sample, but the growth of TB bacilli on traditional solid medium requires 4-8 weeks and consequently delays appropriate treatment in the absence of a confirmed diagnosis. ${ }^{15}$ In the present study, the Gene Xpert test detected the TB causative agent in 36 of 41 pulmonary specimens and 51 of 54 extrapulmonary specimens of confirmed culture positive specimens. Statistically, analyses performed separately for pulmonary and extrapulmonary TB specimens (Table 5), which detected no significant differences between the GeneXpert results and the culture results among patients with pulmonary TB $(P=$ $0.727)$, and patients with extrapulmonary TB $(P=0.344)$. The total agreement among patients with pulmonary TB was $83.33 \%$, and $85.07 \%$ among patients with extra-pulmonary TB. Out of 115 TB specimens, 97 (84.3\%) were GeneXpert positive. Eight samples were found to be culture positive and GeneXpert negative. The possible reason for false negative results could be PCR inhibitors present in the sputum, as the assay appears to be relatively resistant to these PCR inhibitors. The sensitivity of the GeneXpert test, which was as rapid as the AFB staining method, was found to be significantly higher than that of smear in the present study. Data from this study showed that the sensitivity of the GeneXpert assay for smear-positive and smear-negative was $91.6 \%$. However, the specificity was low (50\%) compared to other similar studies. Data showed that the sensitivity of the GeneXpert test for pulmonary specimens was statistically higher $(73.20 \%)$ than that for extrapulmonary specimens (66.70\%) $(P \leq 0.001)$. Our findings on GeneXpert performance are agreed well with other researchers ${ }^{27-29}$ regarding the accuracy of the assay for detection of the presence of TB pathogen bacilli in AFB negative specimens; they reported sensitivities ranged from $72 \%-90 \%$. In view of these data, GeneXpert assay provides a superior close-to-patient test for identifying MTB from the patient's sputum in comparison to conventional bacterial methods. Our data showed that 10 out of 115 were GeneXpert positive but failed to grow any organisms in culture, this could be reasoned as laboratory failure to isolated mycobacteria or due to non-culturable strains of M. tuberculosis, as previously reported. ${ }^{30}$ They reported that the reason could be the synthesis of some intra- or extracellular factors leading to the cessation of cell division. Interestingly, we identified that the previously treated TB patients were more likely to give negative culture results, as the method relies on the viable tubercle bacilli in the samples. The previously exposed to anti-TB drug more likely to reduce bacterial density and also weaken the activity of bacteria in the samples. ${ }^{31}$ Despite being undetected by culture methods, the dead or weakened tubercle bacilli would be identified by molecular diagnostics, which may appear to be an important factor affecting the lower recovery rate by culture. ${ }^{32}$ Even though AFB smear can detect dead cells, the assay requires 6000 to 10,000 organisms per $\mathrm{mL}$ of sample to register as a positive case. ${ }^{33}$ More researches should be done to investigate the contribution of dormant bacteria in lowering the rate of the bacteria from samples collected from previously 
treated TB patients.

\section{Conclusion}

GeneXpert provides a superior diagnostic test for identifying MTB compared to conventional bacteriological methods, although it may give some small false positive results. The main disadvantage of AFB smear is low sensitivity, and the important benefits of GeneXpert assay are early laboratory confirmation of TB disease, earlier treatment initiation; and thus improved patient outcomes. Although culture remains the gold standard for laboratory confirmation of TB disease, GeneXpert should become standard practice for patients suspected of having TB, and all clinicians and public health TB programs should have access to molecular testing for TB to shorten the time of diagnosis.

\section{Competing interests}

The author declares no competing interests.

\section{References}

1. World Health Organization. Global tuberculosis report 2017. World Health Organization; 2017. (Accessed February 12, 2018, at http:// www.who.int/tb/publications/global_report/en/).

2. Huang SY, Chang JR, Liao YC, Dou HY, Chuang MC. Simultaneous detections of genetic fragment and single nucleotide mutation with a three-tiered output for tuberculosis diagnosis. Analytica Chimica Acta 2018; 1007:1-9.

3. Houben RM, Dodd PJ. The global burden of latent tuberculosis infection: a re-estimation using mathematical modelling. PLoS Medicine 2016; 13(10):e1002152.

4. Munir MK, Rehman S, Iqbal R, Saeed MS, Aasim M. Comparison of Gene Xpert MTB/RIF Assays with Conventional Standard Proportion Method for Determination of Drug Susceptibility In multidrug resistant TB suspects. AKEMU 2018; 24(1):570-6.

5. Ahmed ST. Genexpert MTB/RIF assay: A major milestone for diagnosing mycobacterium tuberculosis and rifampicin-resistant cases in pulmonary and extra pulmonary specimens. Infect Dis Prev Control 2018; 1:1.

6. Van Rie A, Enarson D. XDR tuberculosis: an indicator of public-health negligence. The Lancet 2006; 368(9547):1554-6.

7. Mohapatra D, Bhatia V. Gene Xpertmtb/rif: the evidence based path breaking diagnostic tool fortuberculosis diagnosis. Int J Scie Res 2018; $7(8)$.

8. Iram S, Zeenat A, Hussain S, Yusuf NW, Aslam M. Rapid diagnosis of tuberculosis using Xpert MTB/RIF assay-Report from a developing country. Pak J Med Sci 2015; 31:105.

9. Zhang Q, Sun $B Q$, Liu C, Su AN, Wang $X H$, et al. GeneXpert MTB/RIF for rapid diagnosis and rifampin resistance detection of endobronchial tuberculosis. Respirology 2018; 23:950-5.

10. Centers for Disease Control and Prevention (CDC). Updated guidelines for the use of nucleic acid amplification tests in the diagnosis of tuberculosis. MMWR. Morb Mortal Wkly Rep 2009; 58(1):7.

11. Palomino JC. Molecular detection, identification and drug resistance detection in Mycobacterium tuberculosis. FEMS Immunol Med Microbiol 2009; 56(2):103-11.

12. El-Hajj HH, Marras SA, Tyagi S, Kramer FR, Alland D. Detection of Rifampin Resistance in Mycobacterium tuberculosis in a Single Tube with Molecular Beacons. J Clin Microbiol 2001; 39(11):4131-7.

13. Lawn SD, Nicol MP. Xpert MTB/RIF assay: development, evaluation and implementation of a new rapid molecular diagnostic for tuberculosis and rifampicin resistance. Future Microbiol 2011; 6(9):1067-82.

14. Amin B. Diagnostic Assessment of XPERT MTB/ RIF in a sample of mycobacterium tuberculosis in Iraqi Kurdistan patients. Int $\mathrm{J}$ Tech Res App 2015; 3(5):255-9.

15. Zeka AN, Tasbakan S, Cavusoglu C. Evaluation of the GeneXpert MTB/RIF assay for the rapid diagnosis of tuberculosis and detection of RIF-resistance in pulmonary and extrapulmonary specimens. J Clin Microbiol 2011; 28:5434.

16. Helb D, Jones M, Story E, Boehme C, Wallace E, Ho K, et al. Rapid detection of Mycobacterium tuberculosis and rifampin resistance by use of on-demand, near-patient technology. J Clin Microbiol 2010; 48(1):229-37.

17. Banada PP, Sivasubramani SK, Blakemore R, Boehme C, Perkins MD, Fennelly $\mathrm{K}$, et al. Containment of bioaerosol infection risk by the Xpert MTB/RIF assay and its applicability to point-of-care settings. J Clin Microbiol 2010; 48(10):3551-7.

18. Riello FN, Brígido RT, Araújo S, Moreira TA, Goulart LR, Goulart IM. Diagnosis of mycobacterial infections based on acid-fast bacilli test and bacterial growth time and implications on treatment and disease outcome. BMC Inf Dis 2016; 16(1):142.

19. García-Rodríguez JF, Álvarez-Díaz H, LorenzoGarcía MV, Marino-Callejo A, Fernández-Rial Á, Sesma-Sánchez P. Extrapulmonary tuberculosis: epidemiology and risk factors. Enfermlnfecc Microbiol Clin 2011; 29(7):502-9. 
20. Rani P, Bilolikar AK, Sarma VC, Udayasri B, Reddy SG, Kakarla PL, et al. A comparative study of AFB smear and GeneXpert MTB/ RIF assay in pulmonary and extrapulmonary specimens and detection of rifampicin resistance in a tertiary care hospital. J Med Sci Res 2017; 4:115-20.

21. Shi J, Dong $W$, Ma $Y$, Liang $Q$, Shang $Y$, Wang F, et al. GeneXpert MTB/RIF Outperforms Mycobacterial Culture in Detecting Mycobacterium tuberculosis from Salivary Sputum. Bio Med Res Int 2018; 2018:1514381.

22. Pantoja A, Fitzpatrick C, Vassall A, Weyer K, Floyd K. Xpert MTB/RIF for diagnosis of TB and drug-resistant TB: a cost and affordability analysis. Eur Respir J 2013; 42(3):708-20.

23. Laserson KF, Yen NT, Thornton CG, Mai VT, Jones W, An DQ, et al. Improved sensitivity of sputum smear microscopy after processing specimens with C18-carboxypropylbetaine to detect acid-fast bacilli: a study of United States-bound immigrants from Vietnam. J Clin Microbiol 2005; 43(7):3460-2.

24. Kanaujia GV, Lam PK, Perry S, Brusasca PN, Catanzaro A, Gennaro ML. Integration of microscopy and serodiagnostic tests to screen for active tuberculosis. Int $\mathrm{J}$ Tuberc Lung Dis 2005; 9(10):1120-6.

25. Frimpong EH, Adukpo R, Owusu-Darko K. Evaluation of two novel Ziehl-Neelsen methods for tuberculosis diagnosis. West Afr J Med 2005; 24(4):316-20.

26. Lee JS, Kim EC, Joo SI, Lee SM, Yoo CG, Kim $\mathrm{YW}$, et al. The incidence and clinical implication of sputum with positive acid-fast bacilli smear but negative in mycobacterial culture in a tertiary referral hospital in South Korea. J Korean Med Sci 2008; 23(5):767-71.

27. Boehme CC, Nabeta P, Hillemann D, Nicol MP, Shenai S, Krapp F, et al. Rapid molecular detection of tuberculosis and rifampin resistance. New Eng J Med 2010; 363(11):1005-15.

28. Hillemann D, Rüsch-Gerdes S, Boehme C, Richter E. Rapid molecular detection of extrapulmonary tuberculosis by automated GeneXpert MTB/RIF system. J Clin Microbiol 2011; 49(4):1202-5.

29. Moure R, Muñoz L, Torres M, Santin M, Martín $\mathrm{R}$, Alcaide F. Rapid detection of Mycobacterium tuberculosis complex and rifampin resistance in smear-negative clinical samples by use of an integrated real-time PCR method. J Clin Microbiol 2011; 49(3):1137-9.

30. Salina EG, Vostroknutova GN, Shleeva MO, Kaprelyants AS. Cell-cell interactions during the formation and reactivation of "nonculturable" mycobacteria. Microbiology 2006; 75(4):432-7.

31. Zumla A, Nahid P, Cole ST. Advances in the development of new tuberculosis drugs and treatment regimens. Nat Rev Drug Discov 2013; 12(5):388.
32. Nikolayevskyy V, Miotto P, Pimkina E, Balabanova $Y$, Kontsevaya, Ignatyeva $O$, et al. Utility of propidiummonoazide viability assay as a biomarker for a tuberculosis disease. Tuberculosis 2015; 95(2):179-85.

33. Ali AA, Razzaq MA, AL-Saadi MA. Incidence of tuberculosis in Babylon Province-Iraq. Int J Curr Microbiol App Sci 2013; 2(9):1-4. 